\section{¿Es suficiente la protección otorgada por gafas plomadas en cardiología intervencionista?}

\author{
CLAUDIO ZETT-LOBOS ${ }^{1, \mathrm{a}}$, FELIPE VERA-MUÑOZ ${ }^{\text {, a }}$, \\ KATERINA ARRIOLA-ALVAREZ ${ }^{\text {a }}$, OSCAR DÍAZ-RAMOS ${ }^{6, a}$, \\ JORGE GAMARRA ${ }^{5, b, d}$, CRISTIAN FERNÁNDEZ-PALOMO ${ }^{7, a, d}$, \\ LORENZO MERELLO ${ }^{3, \mathrm{f}, 4}$, ALEX D. MORA $^{4, \mathrm{a}}$, \\ ALEJANDRO GUTIÉRREZ ${ }^{2, a}$, MÓNICA CATALÁN-REYES ${ }^{8, c, e}$, \\ SERGIO RAMOS-AVASOLA 2,a,4
}

\section{Protection against ionizing radiation by leaded glass googles during interventional cardiology}

Background: It is not known whether leaded glass goggles with $0.25 \mathrm{~mm} \mathrm{~Pb}$ equivalency, used in interventional cardiology procedures, attenuate radiation below the levels established by the latest recommendation of the International Commission on Radiological Protection (ICRP). Aim: To assess if the degree of attenuation of the secondary ionizing radiation achieved by the use of $0.25 \mathrm{~mm}$ $\mathrm{Pb}$ leaded glass goggles, in occupationally exposed workers in interventional cardiology procedures, meets the latest ICRP recommendations. Material and Methods: A prospective investigation was carried out to compare the eye exposure to secondary ionizing radiation received by occupationally exposed personnel in a 9 months period. A set of two thermo luminescent dosimeters was arranged in the front and back of leaded glass goggles in a cohort of seven members of an interventional cardiology service, exposed to 1057 consecutive procedures. Results: The monthly dose equivalent measurement performed in front of the goggles ranged between 1.1 and $6.5 \mathrm{mSv}$, for paramedics and interventional cardiologists. The radiation measured in the back of the glass varied between 0.66 and $2.75 \mathrm{mSv}$, respectively. The degree of attenuation of the dose at eye level ranged from $40 \%$ to $57.7 \%$, respectively. The projected annual exposure would reach $33 \mathrm{mSv}$ for the interventional cardiologist. Conclusions: With a similar load of work and wearing $0.25 \mathrm{~mm}$ Pb equivalent glass goggles, interventional cardiologists will exceed the crystalline equivalent dose limit recommended by the ICRP (20 mSv/year averaged over the past 5 years $)$.

(Rev Med Chile 2013; 141: 63-69).

Key words: Lens, Crystalline; Radiation dosage; Radiology, Interventional.

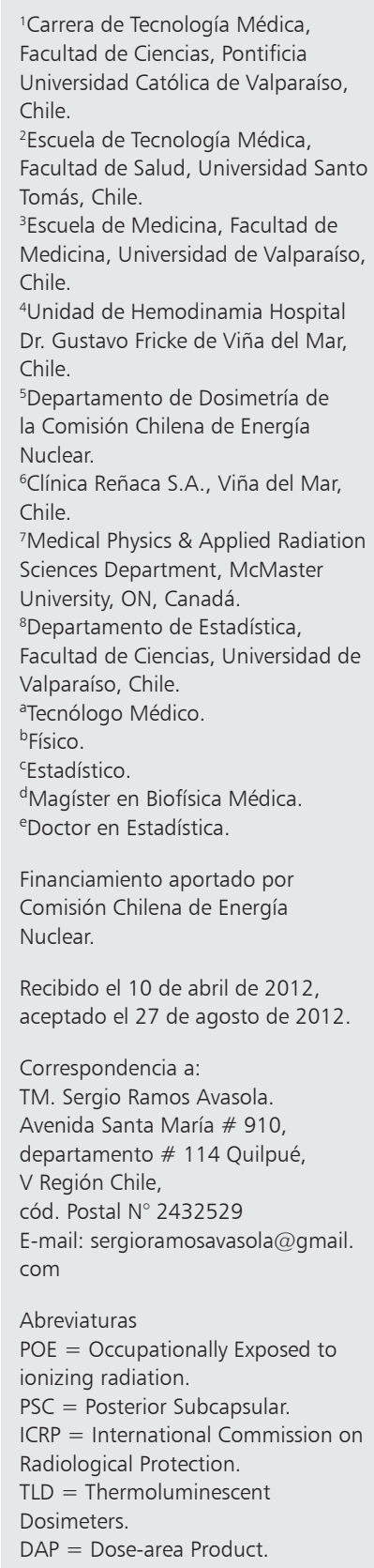

Financiamiento aportado por Comisión Chilena de Energía Nuclear.

Recibido el 10 de abril de 2012 , aceptado el 27 de agosto de 2012.

Correspondencia a:

TM. Sergio Ramos Avasola.

Avenida Santa María \# 910,

departamento \# 114 Quilpué,

$\checkmark$ Región Chile,

cód. Postal № 2432529

E-mail: sergioramosavasola@gmail. com

\section{Abreviaturas}

$\mathrm{POE}=$ Occupationally Exposed to ionizing radiation.

PSC $=$ Posterior Subcapsular.

ICRP = International Commission on

Radiological Protection.

TLD $=$ Thermoluminescent

Dosimeters.

DAP $=$ Dose-area Product.

S e conoce que la opacificación del cristalino es una de las más frecuentes causas de daño a la visión y ceguera a nivel mundial ${ }^{1}$, y de acuerdo a su ubicación anatómica, puede ser clasificada en tres tipos: nuclear, cortical y posterior subcapsular (PSC); esta última ubicación se ha re- lacionado con la exposición a radiación ionizante.

La formación de cataratas causadas por la radiación ocurre en las zonas germinativas, específicamente en la superficie anterior donde las células que se dividen forman una fibra-proteica cristalina que migra hacia el polo posterior del cristalino, la 
Protección por gafas plomadas en cardiología intervencionista - C. Zett-Lobos et al

región posterior subcapsular. La radiación causa a ese nivel un daño directo y además, mediante mecanismos oxidativos, causa el quiebre de la molécula de $\mathrm{ADN}$, migración de células aberrantes y provoca complejos cambios bioquímicos que dan como resultado proteínas cristalinas aberrantes que provocan finalmente una desregulación de la morfología celular del cristalino ${ }^{2-4}$.

Entre los estudios que avalan la asociación entre lesiones de la PSC del cristalino en el personal expuesto a radiación irrizante y los niveles de radiación ionizante secundaria generada en unidades de Cardiología Intervencionista figura un estudio dirigido por Vano, $2010^{5}$, que reportó un riesgo relativo de opacidad de la PSC de cardiólogos intervencionistas igual a 3,2 (38\% comparado con $12 \% \mathrm{p}<0,005)$. Otro estudio dirigido por Ciraj-Bjelac, $2010^{6}$, mostró la misma relación, con un $\mathrm{RR}=5,7(52 \%$ comparado con $9 \% \mathrm{p}<0,005)$ para cardiólogos y un $\mathrm{RR}=5,0$ (45\% comparado con $9 \% p<0,005)$ para enfermeras y técnicos paramédicos. Sin embargo, un estudio publicado el año $2008^{7}$ en una cohorte de 35.705 tecnólogos médicos de los Estados Unidos de Norteamérica seguida por un período de 10 años no arrojó la misma relación causal con un $\mathrm{OR}=1,18$ (IC al 95\% 0,99-1,40). Pero este estudio no analizó los resultados del subgrupo de tecnólogos médicos que trabajan en los departamentos de Cardiología Intervencionista o Radiología Intervencionista, los cuales están expuestos a las más altas dosis de radiación ionizante ${ }^{8,9}$.

La Comisión Internacional de Protección Radiológica (ICRP) emitió el año 2011 una recomendación, en espera de su aprobación, sobre el umbral de dosis absorbida que sugiere 0,5 Gy para el cristalino y para exposición ocupacional la ICRP ahora sugiere una dosis equivalente máxima en el cristalino de $20 \mathrm{mSv} /$ año promediada en un período de 5 años consecutivos, y si se considera sólo un año, la dosis no debiera ser mayor a $50 \mathrm{mSv}^{10}$.

Este nuevo umbral establecido por la ICRP, junto con reportes aislados de cataratas en unidades de Cardiología Intervencionista no optimizadas desde el punto de vista de protección radiológica ${ }^{11}$ y los reportes de una mayor incidencia de cáncer cerebral en cardiólogos intervencionistas ${ }^{12}$, hacen recomendable poner atención a la efectividad de los elementos de protección radiológica; entre ellos los menos evaluados son las gafas plomadas.

El propósito de este estudio es comprobar si la utilización continuada de gafas plomadas de $0,25 \mathrm{~mm} \mathrm{~Pb}$ por el POE en condiciones reales de trabajo de unidades de cardiología intervencionista, otorga una protección acorde con la actual recomendación de la Comisión Internacional de Protección Radiológica.

\section{Material y Métodos}

Este estudio cumple con los estándares internacionales sobre investigación en humanos y fue aprobado por el Comité de Ética del Hospital Dr. Gustavo Fricke de Viña del Mar. Se condujo un estudio prospectivo, que involucró una cohorte de siete miembros POE en una Unidad de Hemodinamia, que estuvieron expuestos a una fuente de radiación ionizante secundaria generada en 1.057 procedimientos cardiológicos intervencionistas, 763 angiografías coronarias y 294 angioplastías coronarias. Este fue llevado a cabo en un Hospital Base de nivel primario de la $\mathrm{V}$ región de Chile, desde febrero a noviembre del año 2007. El equipo utilizado fue un angiógrafo marca Siemens, modelo Axiom Artis, año 2005 (Siemens, Munich, Alemania), equipado con un panel plano para estudios cardiacos. Al momento de la realización de cada procedimiento angiográfico había en el pabellón un cardiólogo intervencionista, un técnico paramédico, un tecnólogo médico y una enfermera, los cuales estaban distribuidos en el pabellón de acuerdo a la Figura 1. Todos ellos debidamente acreditados con Cursos de Radioprotección.

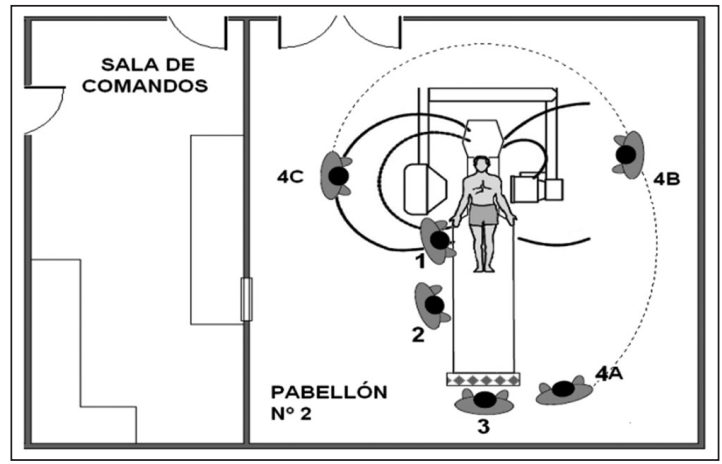

Figura 1. $1=$ Médico intervencionista, $2=$ Técnico paramédico, 3= Tecnólogo médico, 4= Enfermera (Imagen cedida gentilmente por MSc. Cristian Fernández Palomo. Tesis de pregrado "Detección y Medición de Radiación Ionizante Secundaria Generada por un Equipo de Angiografía Coronaria" de la Escuela de Tecnología Médica, Universidad de Valparaíso, 2006). 
Al momento de los exámenes cada uno de los integrantes del POE utilizó elementos de protección radiológica que eran personales e individuales que consistió de 7 delantales plomados y 7 protectores tiroideos con una equivalencia de $0,5 \mathrm{~mm} \mathrm{~Pb}$. Las 7 gafas plomadas utilizadas que fueron idénticas entre sí, fueron adquiridas el año 2004 a un único proveedor, poseían sólo protección frontal con una equivalencia de $0,25 \mathrm{~mm} \mathrm{~Pb}$ y junto con las medidas anteriores, también se utilizó en cada examen una pantalla de vidrio plomado suspendida del techo y un faldón plomado bajo la camilla de examen con una equivalencia de $0,5 \mathrm{~mm} \mathrm{~Pb}$.

En relación a los parámetros dependientes del angiógrafo, la fluoroscopia fue pulsada con 15 pulsos/segundo, la cinecoronariografía se realizó con 15 imágenes /segundo y la colimación de las adquisiciones fue realizada en forma habitual.

\section{Mediciones}

Las dosis equivalentes a nivel del cristalino fueron medidas en $\mathrm{mSv}$ con dos dosímetros termoluminicentes (TLD) $\mathrm{LiF}(\mathrm{Mg}$; $\mathrm{Ti}$ ) por persona dispuestos de manera frontal y posterior al vidrio

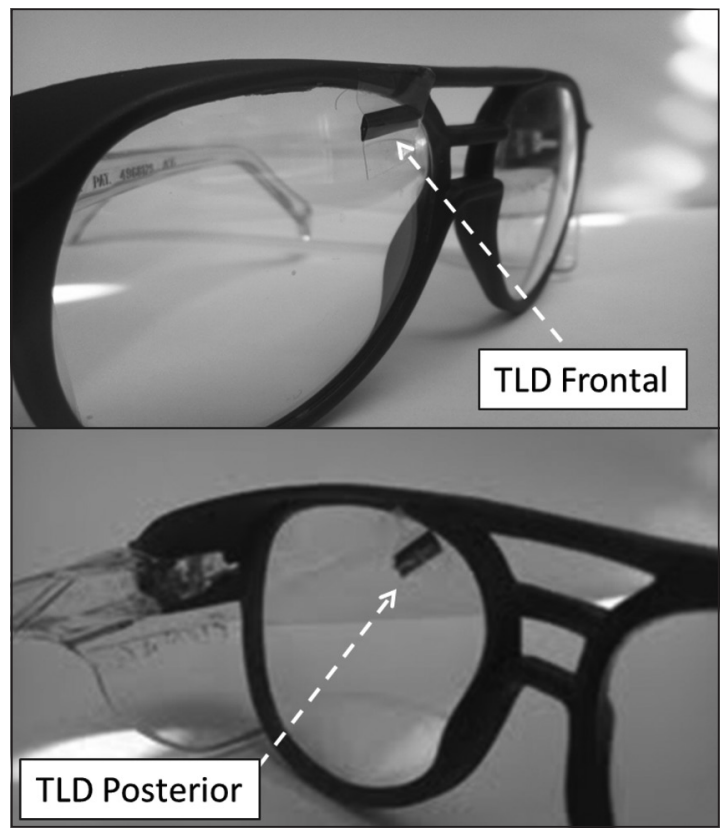

Figura 2. Imagen cedida gentilmente por alumnos. Arriola K., Vera F. y Zeth C. Tesis de pregrado "Evaluación del Cristalino con Imágenes Scheimpflug de Pentacam en Personal Ocupacionalmente Expuesto a Radiación Ionizante Secundaria en Unidades de Hemodinamía y Angiografía Coronaria" de la Escuela de Tecnología Médica, Universidad de Valparaíso, 2007). plomado de las gafas como se puede apreciar en la Figura 2, fueron proporcionados por el laboratorio de dosimetría de la Comisión Chilena de Energía Nuclear (CCHEN), Certificada bajo la ISO 9001:2008 y que cumple las exigencias del Organismo Internacional de Energía Atómica.

Una vez instalados los TLDs, estos fueron portados por los miembros del POE por períodos de un mes, tras el cual fueron reemplazados por nuevos dosímetros termoluminicentes (TLD) TLDs. Los TLDs usados fueron medidos en un lector automático de TLDs Harshaw modelo 5500 (Thermo Scientific, Massachusetts, Estados Unidos de Norteamérica y posteriormente regenerados para ser nuevamente utilizados.

\section{El Producto dosis-área}

El producto dosis/áerea de cada paciente fue obtenida de la cámara de ionización interna (Diametor, Freiburg, Alemania), con certificados de calibración PTW Freiburg Nº 045902 y 045745 de agosto de 2004; que está integrada a la salida del tubo de rayos- $\mathrm{X}$ y es periódicamente chequeada por el Servicio Técnico de Siemens Chile, quienes constatan que los valores de tolerancia de la cámara de ionización no excedan de $2 \%$ del valor de fábrica (Anexo $\mathrm{N}^{\circ} 1$ ).

\section{Análisis estadístico}

El análisis estadístico fue desarrollado utilizando un software estadístico (SPSS, Chicago, IL; EE.UU. versión 17). En el caso de aquellos estamentos con más de una persona (Médicos y Tecnólogos Médicos) se promediaron sus valores, los datos continuos se expresaron como la media \pm desviación estándar (SD) y los datos categóricos son expresados en porcentaje y su frecuencia absoluta. Dada la falta de normalidad se utilizó la prueba de Kruskall-Wallis para las comparaciones. El análisis estadístico está limitado por el hecho que la dosis acumulativa mensual fue establecida con dosimetría, y por lo tanto, el promedio de dosis absorbida por examen solamente puede ser extrapolado, pero no medido directamente. El nivel de significancia estadística utilizado fue de un $\mathrm{p}<0,05$.

\section{Resultados}

El POE al momento del estudio estuvo conformado por 5 hombres y dos mujeres con un 
Protección por gafas plomadas en cardiología intervencionista - C. Zett-Lobos et al

promedio de edad de 41,5 $\pm 6,2$ años, del total, 3 eran médicos, el resto pertenecía al estamento no médico (tecnólogos médicos, enfermeras y técnicos paramédicos). La experiencia de los médicos intervencionistas era en promedio superior a los 15 años.

En cuanto a los exámenes realizados, estos incluyeron 763 angiografías coronarias (DAP $=1797$ $\pm 860 \mu \mathrm{Gym}^{2}$ ) y 294 angioplastias coronarias (DAP $=5683 \pm 2962 \mu \mathrm{Gym}^{2}$ ), en cuanto a la complejidad de las angioplastias abordadas 15\% correspondió a angioplastías de lesiones en bifurcación, un 10\% a lesiones crónicamente ocluidas y el resto fueron angioplastias clasificadas como tipo A, B1 y B2 según la clasificación del TASK FORCE ACC-AHA y el tiempo promedio de fluoroscopia empleado en los exámenes fue de 4,39 min.

En la Tabla 1 se pueden apreciar los niveles de radiación recibida por el POE en cuerpo total y a nivel de cristalino; la dosis equivalente acumulativa promedio mensual obtenida de la zona frontal de las gafas utilizadas por el POE fue de 1,10 hasta 6,50 $\mathrm{mSv}$, en técnico paramédico y cardiólogo respectivamente. Al extrapolar estos datos a dosis anuales se proyecta una dosis de $13,2 \mathrm{mSv}$ y 78 $\mathrm{mSv}$. Esto corresponde al $66 \%$ y $390 \%$ respectivamente de la última recomendación para la dosis promedio límite anual máxima para el ojo (20
mSv, ICRP).

La dosis equivalente acumulativa promedio mensual mínima y máxima medida en la zona posterior de las gafas, fue de 0,66 a 2,75 $\mathrm{mSv}$, en técnico paramédico y cardiólogo respectivamente. $\mathrm{Al}$ extrapolar estos datos a dosis anuales se proyecta una exposición de 7,9 mSv a $33 \mathrm{mSv}$. Esto corresponde al $39,5 \%$ y $165 \%$ de la última recomendación para la dosis promedio límite anual para el ojo (20 mSv, ICRP).

El grado de atenuación de los Rayos-X incidentes sobre las gafas fluctuó entre un 40 y 57,7\%, resultados que corresponden al técnico paramédico y cardiólogo respectivamente.

En la Figura 3, se muestra una comparación entre las dosis promedio por examen en $\mu \mathrm{Sv}$ según el tipo de integrante del staff presente en los procedimientos de Cardiología Intervencionista, la enfermera recibe en el cristalino 3,8 veces menos dosis equivalente que la que recibe el médico cardiólogo.

En la Tabla 2, se aprecia la relación dosis/ /DAP en el cristalino para cada uno de los integrantes del POE que están en cada procedimiento, ésta difiere significativamente entre cada uno de los distintos estamentos de profesionales evaluados $(\mathrm{p}<0,001)$, correspondiendo el mayor valor al estamento médico con un valor de $12 \mu \mathrm{Sv} / 1000$

Tabla 1. Comparación de dosis promedio recibidas en los TLDs ubicados frontal y posterior al cristal de las gafas plomadas equivalentes a 0,25 $\mathrm{mm}$ de $\mathrm{Pb}$

\begin{tabular}{|c|c|c|c|c|}
\hline & $\begin{array}{l}\text { Dosímetros* de } \\
\text { cuerpo total }(\mathrm{mSv}) \\
\text { Promedio }\left(\mathrm{CV}^{*}\right)\end{array}$ & $\begin{array}{c}\text { TLD } \\
\text { Frontal (mSv) } \\
\text { Promedio (CV) }\end{array}$ & $\begin{array}{c}\text { TLD } \\
\text { Posterior (mSv) } \\
\text { Promedio (CV) }\end{array}$ & $\begin{array}{c}\% \text { de } \\
\text { atenuación }\end{array}$ \\
\hline \multicolumn{5}{|l|}{ Cardiólogo intervencionista } \\
\hline Dosis en cuerpo total mensual & $0,82(29,8)$ & & & \\
\hline Dosis acumulada mensual en gafas & & $6,50(6,2)$ & $2,75(7,4)$ & 57,7 \\
\hline \multicolumn{5}{|l|}{ Tecnólogo médico } \\
\hline Dosis en cuerpo total mensual & $0,14(194)$ & & & \\
\hline Dosis acumulada mensual en gafas & & $2,10(23,3)$ & $1,01(24,4)$ & 52,0 \\
\hline \multicolumn{5}{|l|}{ Enfermera } \\
\hline Dosis en cuerpo total mensual & $0,23(9,2)$ & & & \\
\hline Dosis acumulada mensual en gafas & & $1,7(4,8)$ & $0,94(6,5)$ & 44,7 \\
\hline \multicolumn{5}{|l|}{ Técnico paramédico } \\
\hline Dosis en cuerpo total mensual & $0,11(96)$ & & & \\
\hline Dosis acumulada mensual en gafas & & $1,10(14,8)$ & $0,66(4,9)$ & 40,0 \\
\hline
\end{tabular}

*Dosímetro fílmico utilizado debajo del delantal plomado. *CV: Coeficiente de variación. 


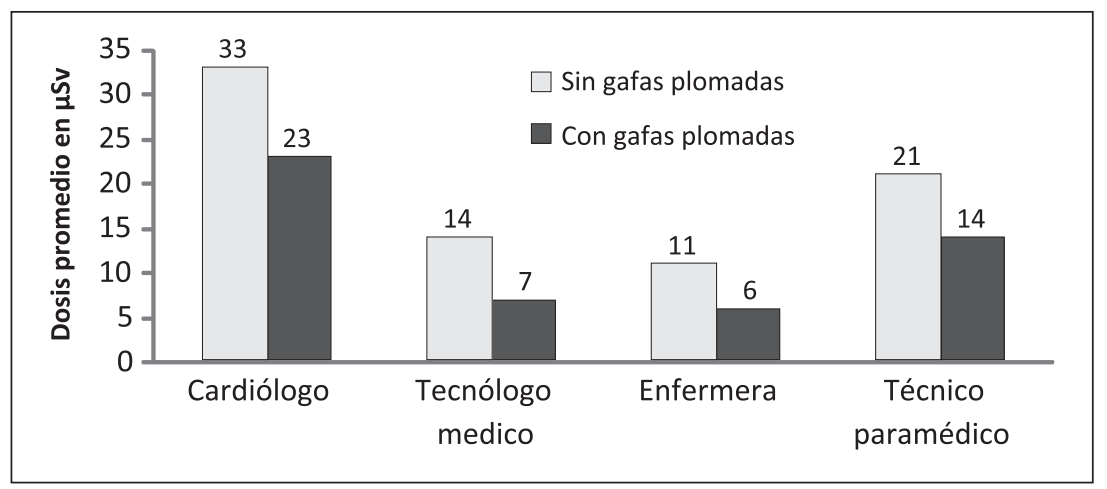

Figura 3. Dosis promedio por examen registrada en dosímetro frontal y posterior a gafas plomadas según estamento clínico. Dosis equivalente promedio por examen en el cristalino. Los valores son presentados para cada uno de los profesionales que conforman el staff de un procedimiento intervencional cardiológico. Las barras muestran las dosis registradas por los TLDs anterior y posterior a las gafas plomadas.

Tabla 2. Comparación delarelación dosis/DAP y las dosis a nivel del ojo en el POE que participa en procedimientos de cardiología intervencionista

\begin{tabular}{lccccc}
\hline & Cardiologo & $\begin{array}{c}\text { Tecnologo } \\
\text { medico }\end{array}$ & $\begin{array}{c}\text { Enfermera } \\
\text { paramedico }\end{array}$ & $\begin{array}{c}\text { Tecnico } \\
\text { bilateral* }\end{array}$ \\
\hline $\begin{array}{l}\text { DAP acumulado mensual } \\
\left.\text { (Gy cm }{ }^{2}\right)\end{array}$ & 271 & 34 & 46 & 90 \\
$\begin{array}{l}\text { Dosis promedio en Cristalino por examen } \\
\text { ( } \mu \mathrm{Sv})\end{array}$ & 33 & 14 & 11 & 21 \\
$\begin{array}{l}\text { Relacion dosis/DAP en cristalino } \\
\left(\mu \mathrm{Sv} / 1000 \mathrm{cGycm}{ }^{2} \text { ) }\right.\end{array}$ & 12,0 & 5,1 & 4,0 & 7,6 & $<0,001$ \\
\end{tabular}

* Tests de Kruskall-Wallis.

$\mathrm{cGycm}^{2}$ y el menor valor se verificó en el estamento de la enfermera con un valor de $4,0 \mu \mathrm{Sv} / 1000 \mathrm{cGy}$ $\mathrm{cm}^{2}$; es decir, los médicos a la altura del cristalino reciben 3 veces más radiación de la que recibe la enfermera, lo que además está estrechamente relacionado con la distancia a la fuente de radiación.

\section{Discusión}

Emplear aproximaciones para conocer la dosis recibida en el cristalino mediante la utilización de datos dosimétricos ha sido difícil de establecer ${ }^{13,14}$, por lo tanto, para conocer el valor real de la dosis anual recibida en este órgano es necesario medirla $y$ confrontar sus resultados para verificar que no se excedan los nuevos umbrales de dosis anuales para la exposición ocupacional del cristalino sugeridos por la ICRP.

En cuanto a la normativa nacional, esta es más permisiva y más antigua, de hecho, el límite de dosis anual fue fijada en $300 \mathrm{mSv}$ especificado en el Reglamento de Protección Radiológica.

En este estudio se observó que las dosis equivalentes del cristalino de los cardiólogos intervencionistas medidas delante de las gafas plomadas exceden el límite anual actualmente sugerido por la ICRP con un valor promedio anual de $78 \mathrm{mSv}$, lo que es levemente superior a lo publicado el año 2006 por Maeder M, 2006 ${ }^{15}$, quien reportó un promedio anual de $66,1 \mathrm{mSv}$. Todos los demás estamentos permanecieron bajo el umbral de 50 $\mathrm{mSv}$. Al medir las dosis equivalentes registradas por los TLDs ubicados posterior a gafas se observó una dosis de $33 \mathrm{mSv}$, lo que implica que las gafas logran atenuar la radiación en $57 \%$. Este valor es relevante, puesto que si el estamento de cardiólogos sigue con esa carga de trabajo probablemente excederán el valor promedio de 5 años de dosis máximo sugerido recientemente por la ICRP (20 $\mathrm{mSv})$.

También es llamativo que el estamento de tecnólogos médicos siendo el más alejado, tenga más dosis que la enfermera y el técnico paramédico, 
una probable explicación podría estar dada por el hecho que por lo general el tecnólogo médico en los procedimientos, tiene un ángulo de visión directo y sin obstáculos entre la radiación secundaria proveniente del paciente y sus gafas plomadas, no así el técnico paramédico y la enfermera que habitualmente se encuentran escudados por el médico y/o el vidrio plomado.

La dosis promedio por examen observada en los cardiólogos intervencionistas fue la mayor, con un valor de $33 \mu \mathrm{Sv}$ en el dosímetro ubicado entre el vidrio plomado y el tubo de rayos X. Este valor se encuentra dentro del rango de valores observados en otros estudios ${ }^{15-18}$ que reportaron los siguientes valores promedio por examen: 13, 13, 44 y 294 $\mu \mathrm{Sv}$ respectivamente. El último valor se escapa significativamente de los valores reportados. Pero se debe considerar que ese estudio fue realizado el año 1998 con equipos con intensificador de imagen, los cuales emiten $30 \%$ más de radiación que los actuales equipos con panel plano ${ }^{19}$. Además, en ese estudio el uso de los dispositivos de protección radiológica fue irregular.

El valor promedio entre las dosis a nivel del ojo y la relación dosis/DAP estuvieron en el rango de 4,0 a $12 \mu \mathrm{Sv} / 1000$ cGy cm² (enfermera y cardiólogos respectivamente). La cifra del cardiólogo está de acuerdo con un artículo publicado el año $2010^{16}$ que reportó un valor de $13,7 \mu \mathrm{Sv} / 1000$ cGy $\mathrm{cm}^{2}$, sin embargo, con estudios anteriores no se verificó esta concordancia, pues mostraron valores mayores del orden de: $33-60 \mu \mathrm{Sv} / 1000 \mathrm{cGy} \mathrm{cm}{ }^{218}$; 15-29 $\mu \mathrm{Sv} / 1000 \mathrm{cGy} \mathrm{cm}^{2}$. . Sin embargo, debe hacerse el alcance que uno de esos resultados fue obtenido el año 1998, cuando no aparecía aún la tecnología de paneles planos.

Esto hace imperativo el adecuado uso de estrategias para minimizar las dosis de radiación tanto para el paciente como para el personal que trabaja con estos pacientes, entre las cuales se pueden mencionar las siguientes: emplear fluoroscopia de baja pulsación por segundo, minimizar los tiempos de fluoroscopia, utilizar la menor cantidad de imágenes magnificadas necesarias para una interpretación exacta, minimizar la distancia del paciente al detector de imágenes y el tubo de Rayos-X, optimizar la colimación del haz, disminuir el número de adquisiciones, utilizar valores de $\mathrm{kV}$ lo más alto posible para disminuir en $\mathrm{mA}$, omitir la ventriculografía izquierda si la información diagnóstica está disponible a partir de otros estudios y lo más importante es el empleo de gafas plomadas con una mayor equivalencia de plomo $(0,33$ ó $0,5 \mathrm{~mm}$ de $\mathrm{Pb})$ para el personal que trabaja en este tipo de procedimientos ${ }^{20}$.

\section{Conclusiones}

Las dosis equivalentes del POE a nivel del cristalino fueron medidas en procedimientos de cardiología intervencional mediante el empleo de TLD frontal y posterior a las gafas plomadas. En promedio las más altas dosis de los TLDs ubicados en la zona frontal de las gafas se observaron en los cardiólogos, mientras que las menores se verificaron en el estamento de la enfermera. Al extrapolar estos datos a cifras anuales y confrontarlos a los límites fijados por la Normativa Nacional Chilena (150 $\mathrm{mSv}$ ), no habría ningún estamento del POE que los excediera, pero si los confrontamos con la última recomendación de la ICRP referente a la dosis límite anual para el cristalino $(50 \mathrm{mSv})$ se observó que los cardiólogos exceden el límite de exposición con un valor de $78 \mathrm{mSv}$. En contraste, los demás estamentos no exceden dicha recomendación.

El empleo de gafas plomadas con una equivalencia de $0,25 \mathrm{~mm}$ de $\mathrm{Pb}$ logra atenuar la radiación en el caso de los cardiólogos a $33 \mathrm{mSv}$, excediendo todavía la última recomendación de la ICRP de 20 mSv/año como promedio.

Agradecimientos: Agradecemos a la Comisión Chilena de Energía Nuclear por el valioso aporte de TLDs y a las facilidades dadas para el análisis de las

Anexo $\mathbf{N}^{\circ} 1$.

\% de derivación respecto del valor basal de fábrica y fechas de calibración de cámara de ionización Diamentor año 2007

\begin{tabular}{|c|c|}
\hline Fecha de visita & Valor \\
\hline 07- Enero -2007 & 1,07 \\
\hline 12- Junio -2007 & 1,18 \\
\hline
\end{tabular}


dosimetrías en sus laboratorios, también se hacen extensivos los agradecimientos al Ingeniero Sr. José Carrillo del servicio técnico de Siemens Chile por su valiosa asesoría en los temas de calibración y chequeo de la cámara de ionización y finalmente a la Fundación Cardiovascular Dr. Jorge Kaplan por las facilidades logísticas de secretariado e insumos prestados para la realización de este estudio.

\section{Referencias}

1. Rehani M, Vano E, Ciraj-Bjelac O, Kleiman N. Radiation and cataract. Radiat Prot Dosimetry 2011; 147: 300-4.

2. Ainsbury E, Bouffler S, Dorr W, Graw J, Muirhead C, Edwards A, et al. Radiation cataractogenesis: a review of recent studies. Radiat Res 2009; 172: 1-9.

3. Chang P, Bjornstad K, Rosen C, Lin S, Blakely E. Particle radiation alters expression of matrix metalloproteases resulting in ECM remodeling in human lens cells. Radiat Environ Biophys 2007; 46: 187-94.

4. Jones J, McCarten M, Manuel K, Djojonegoro B, Murray J, Feiversen A, et al. Cataract formation mechanisms and risk in aviation and space crews. Aviat Space Environ Med 2007; 78: 56-66.

5. Vano E, Kleiman N, Duran A, Rehani M, Echeverri D, Cabrera M. Radiation cataract risk in interventional cardiology personnel. Radiat Res 2010; 174: 490-5.

6. Ciraj-Bjelac O, Rehani M, Sim K, Liew H, Vano E, Kleiman N. Risk for radiation-induced cataract for staff in interventional cardiology: is there reason for concern? Catheter Cardiovasc Interv 2010 ; 76: 826-34.

7. Chodick G, Bekiroglu N, Hauptmann M, Alexander B, Freedman D, Doody M, et al. Risk of cataract after exposure to low doses of ionizing radiation: a 20 -year prospective cohort study among US radiologic technologists. Am J Epidemiol 2008; 168: 620-31.

8. Rehani M, Ortiz-López P. Radiation effects in fluoroscopically guided cardiac interventions-keeping them under control. Int J Cardiol 2006; 109: 147-51.
9. Valentin J. Avoidance of radiation injuries from medical interventional procedures. Ann ICRP 2000; 30: 7-67.

10. Rehani M, Vano E, Ciraj-Bjelac O, Kleiman N. Radiation and Cataract. Radiat Prot Dosimetry 2011; 147: 300-4.

11. Vano E, González L, Beneytez F, Moreno F. Lens injuries induced by occupational exposure in non-optimized interventional radiology laboratories. Br J Radiol 19981; 71: 728-33.

12. Finkelstein M. Is brain cancer an occupational disease of cardiologists? Can J Cardiol 1998 ; 14: 1385-8.

13. Padovani R, Bernardi G, Malisan M, Vano E, Morocutti G, Fioretti P. Patient dose related to the complexity of interventional cardiology procedures. Radiat Prot Dosimetry 2001; 94: 189-92.

14. Servomaa A, Karppinen J. The dose-area product and assessment of the occupational dose in interventional radiology. Radiat Prot Dosimetry 2001; 96: 235-6.

15. Maeder M, Brunner-La Rocca H, Wolber T, Ammann P, Roelli H, Rohner F, et al. Impact of a lead glass screen on scatter radiation to eyes and hands in interventional cardiologists. Catheter Cardiovasc Interv 2006; 67: 18-23.

16. Efstathopoulos E, Pantos I, Andreou M, Gkatzis A, Carinou E, Koukorava C, et al. Occupational radiation doses to the extremities and the eyes in interventional radiology and cardiology procedures. Br J Radiol 2011; 84: 70-7.

17. Lie O, Paulsen G, Wohni T. Assessment of effective dose and dose to the lens of the eye for the interventional cardiologist. Radiat Prot Dosimetry 2008; 132: 313-8.

18. Vano E, González L, Guibelalde E, Fernández J, Ten JI. Radiation exposure to medical staff in interventional and cardiac radiology. Br J Radiol 1998; 71: 954-60.

19. Tsapaki V, Kottou S, Kollaros N, Dafnomili P, Koutelou $\mathrm{M}$, Vano E, et al. Comparison of a conventional and a flat-panel digital system in interventional cardiology procedures. Br J Radiol 2004; 77: 562-7.

20. Avendaño G, Fernández C. Effect of Changes in Technical Parameters in Radiological Safety. Journal of Physics 2007; 90: 1-9. 